\title{
Transient Anisocoria during Medial Blowout Fracture Surgery
}

Jae Il Lee,

Seok Joo Kang,

Seong Pin Jeon,

Hook Sun

Department of Plastic and Reconstructive Surgery, Busan Baik Hospital, Inje University School of Medicine, Busan, Korea

No potential conflict of interest relevant to this article was reported.

\begin{abstract}
Transient anisocoria is rare during blowout fracture reconstruction. We report a case of transient anisocoria occurring during medial blowout fracture reconstruction and review the relevant literature. A 54-year-old woman was struck in the face and was admitted for a medial blowout fracture of the left eye. During the operation, persistent bleeding occurred. To control this bleeding, a 1\% lidocaine solution with 1:200,000 epinephrine was applied to the orbital wall with cotton pledgets. In total, $40 \mathrm{~mL}$ of local anesthetic was used for the duration of the operation. After approximately three hours of the surgery, the ipsilateral pupil was observed to be dilated, with sluggish response to light. By 3 hours after the operation, the mydriasis had resolved with normal light reflex. In conclusion, neurological and ophthalmologic evaluation must be performed prior to blowout fracture surgery. Preoperative ophthalmic evaluation is simple and essential in ruling out any preexisting neurologic condition. Moreover, surgeons must be aware of the fact that excessive injection of lidocaine with epinephrine for hemostasis during orbital wall surgery can result in intraoperative anisocoria. Anisocoria-related situations must be addressed in a proficient manner through sufficient understanding of the mechanism controlling the pupillary response to various stimuli.
\end{abstract}

Keywords: Anisocoria / Mydriasis / Blow out fracture

\section{INTRODUCTION}

Transient anisocoria is rare during blowout fracture reconstruction. However, occurrence of simple anisocoria or benign pupillary inequality is not uncommon. A new-onset mydriasis is a distressing event for surgeons. Etiologies for pupillary dilation usually include systemic causes, such as neurological and vascular disorders, local causes such as pharmacological effects, and congenital iris disorders. Intraoperative transient anisocoria can be a sign of significant intracranial injury, such as cerebral trauma or ruptured aneurysm. Therefore, appropriate understanding and

\section{Correspondence: Seok Joo Kang}

Department of Plastic and Reconstructive Surgery, Busan Baik Hospital, Inje University School of Medicine, 75 Bokji-ro, Busanjin-gu, Busan 47392, Korea E-mail: sonydr@naver.com

Received March 9, 2016 / Revised July 18, 2016 / Accepted July 20, 2016 diagnosis of anisocoria during surgery are needed, and prompt and correct treatment is essential. The authors report on a case of transient anisocoria during blowout fracture reconstruction and review the relevant literature.

This study was reviewed and approved by the Ethics Review Board of the InJe University Health Center.

\section{CASE REPORT}

A 54-year-old woman who was struck in the face was admitted for a medial blowout fracture of the left orbit. During the preoperative ophthalmic examination, the pupils were equal at $3.5 \mathrm{~mm}$, with a positive light reflex as well as normal optic disc and fundus. General anesthesia was performed using propofol for induction and fentanyl was used as the secondary anesthetic, desflurane as the inhalation anesthetic and rocuronium as the muscle 
relaxant. A local anesthetic mixture of 1:200,000 epinephrine and $1 \%$ lidocaine was injected into the conjunctiva. The orbital wall was accessed via a subciliary incision.

During the operation, persistent bleeding occurred from the anterior and posterior ethmoidal arteries. To control this bleeding, a 1\% lidocaine solution with 1:200,000 epinephrine was applied to the orbital wall with cotton pledgets. In total, $40 \mathrm{~mL}$ of lo$\mathrm{cal}$ anesthetic was used for the duration of the operation. After approximately three hours of the surgery, the ipsilateral pupil was observed to be dilated, with sluggish response to light (Fig. 1). The orbital wall was reconstructed using Medpor (Porex Surgical Inc., Newnan, GA, USA) after extensive hemostasis. The pupillary dilation, though slightly improved, persisted even during the postoperative setting. By 3 hours after the operation, the mydriasis had resolved with normal light reflex. No other specific ophthalmic findings were subsequently observed (Fig. 2).

\section{DISCUSSION}

Mydriasis that occurs suddenly during an operation can be an embarrassment for a surgeon, and can lead to unnecessary examinations or procedures. However, the occurrence of simple

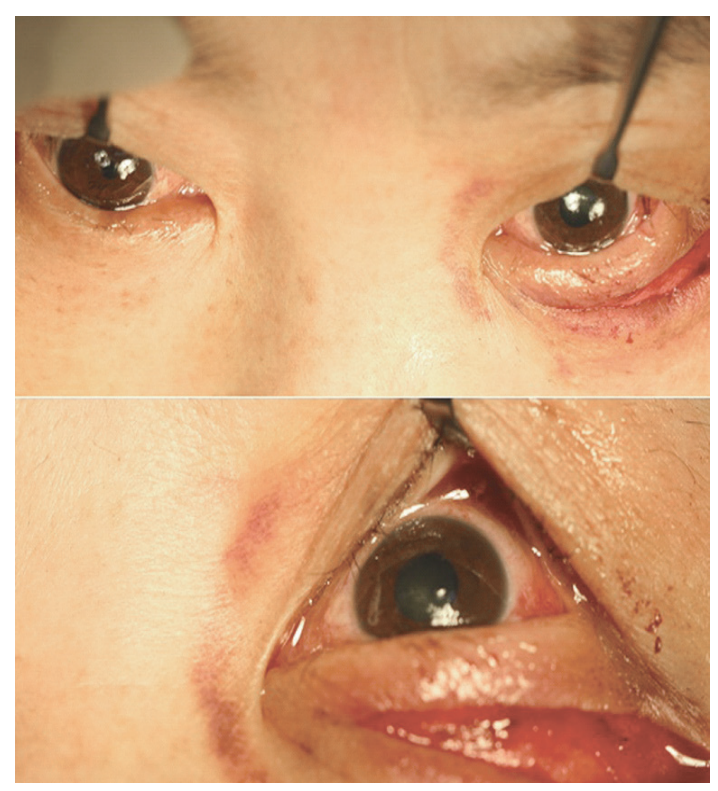

Fig. 1. Intraoperative photograph shows the left eye with dilated pupil. anisocoria or benign pupillary inequality is not uncommon. According to a report by Lim et al. [1], intraoperative mydriasis occurred in $2.1 \%$ of cases involving a subconjunctival approach during orbital floor fracture surgery. Typically, under general anesthesia, pupil size decreases and the light reflex slows or disappears.

Although transient anisocoria may not be directly related to orbital fracture surgery, even incident finding of anisocoria warrants work-up for neurosurgical emergencies. A meticulous neurological examination must be performed prior to any operation, because unilateral mydriasis can be caused by brain lesions such as intracranial hemorrhage, cerebral compression, and expanding aneurysms [2].

During orbital fracture surgery, potential causes for unilateral mydriasis include local anesthetic infiltration into the eyelids, orbit, and paranasal sinuses, manipulation of the inferior branch of the oculomotor nerve, manipulation of the ciliary ganglion, direct injury to the optic nerve, and globe injury [2,3]. Pupillary function is controlled by a balance of sympathetic (dilator) and parasympathetic (constrictor) neural pathways [4]. The parasympathetic fibers originate in the Edinger-Westphal nucleus in the midbrain and follow the oculomotor nerve into the orbit. Once in the orbit,

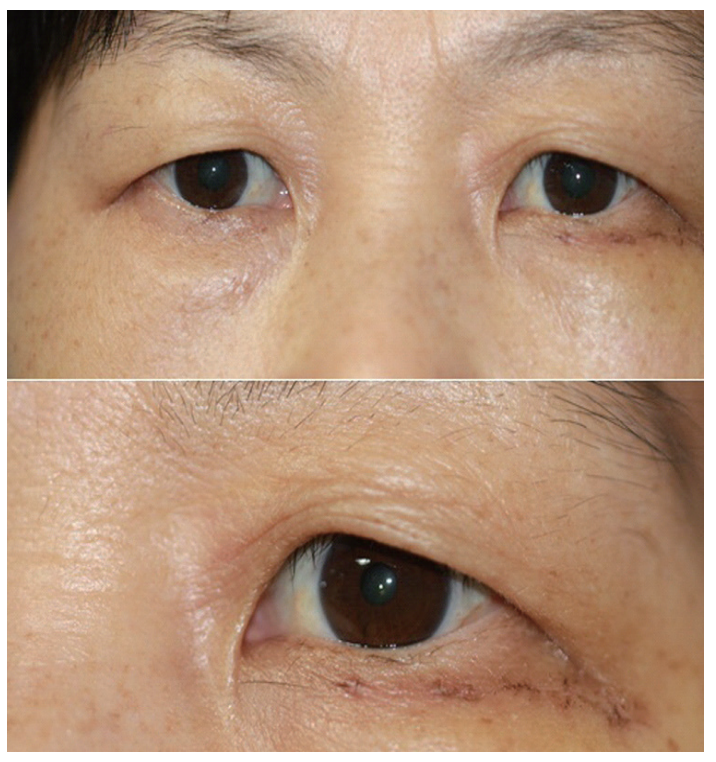

Fig. 2. One week postoperative photograph shows the left eye with normal sized pupil. 
the parasympathetic fibers are located in the inferior branch of the oculomotor nerve and synapse in the ciliary ganglion. Postsynaptic parasympathetic fibers travel in the short ciliary nerves and reach the sphincter pupillae, causing constriction (Fig. 3). As the inferior oblique and inferior rectus muscles share the capsulopalpebral fascia, overly aggressive manipulation of the muscles during exposure of the orbital floor fracture may consequently lead to injury to the ciliary ganglion or the inferior branch of the oculomotor nerve, resulting in mydriasis on rare occasions.

Pharmacologic agents are also known to cause mydriasis. An anesthetic mixture containing lidocaine and epinephrine injected into the conjunctiva or eyelids can diffuse into the iris muscular complex, causing cycloplegia and consequent mydriasis.

In the presented case, the surgeons focused on the drugs used for hemostasis and local anesthesia to be the primary cause for the anisocoria. Relatively rapid onset and resolution of the segmental anisocoria is believed to be due to the action of lidocaine on the small branches of the short ciliary nerves that innervate the constrictor pupillae.

Several factors support the anisocoria to be induced by anesthetic diffusion. During the operation, there was low probability of any direct nerve injury, since the orbital fracture was in the medial wall. The operation required large amounts of local anesthetic mixture for hemostasis; and there was excessive periorbital retraction resulting from an operative time exceeding 3 hours and bleeding during the surgery. Another factor is that the mydriasis appeared 3 hours after the start of operation with the involved pupil returning to normal size approximately 3 hours after the completion of surgery.

Therefore, it was determined that these factors led to the appearance of transient, intraoperative unilateral mydriasis.

In conclusion neurological and ophthalmologic evaluation must be performed prior to blowout fracture surgery. Preoperative ophthalmic evaluation is simple and essential in ruling out any preexisting neurologic condition [5]. Moreover, surgeons must be aware of the fact that excessive injection of lidocaine with epinephrine for hemostasis during orbital wall surgery can result in intraoperative anisocoria. Anisocoria-related situations must be addressed in a proficient manner through sufficient understanding of the mechanism controlling the pupillary response to various stimuli.

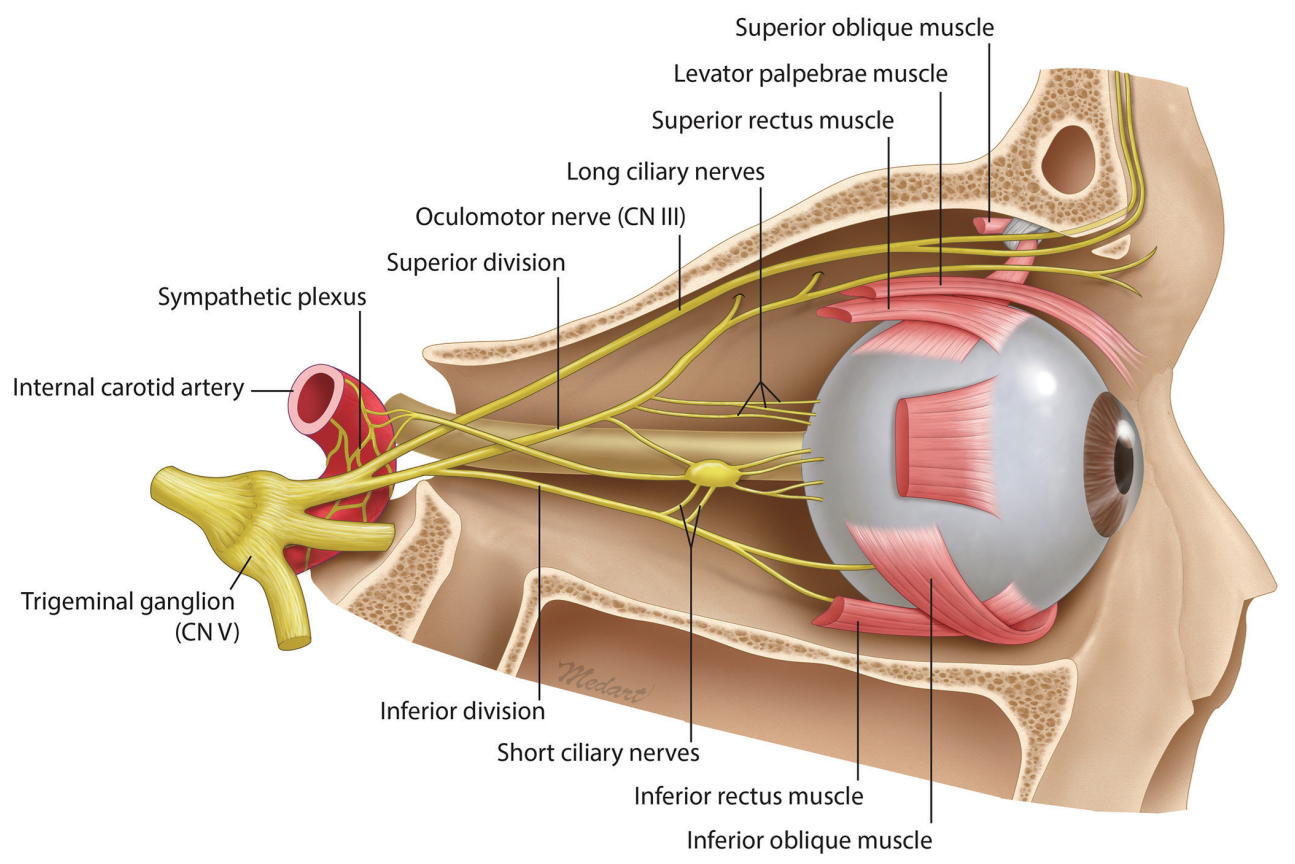

Fig. 3. Illustration of ciliary ganglion and innervations to the muscles of the eye. 


\section{REFERENCES}

1. Lim TC, Tan WT, Lim J, Cheng LC. Where's the point? Plast Reconstr Surg 1998;101:861-2.

2. Yeo MS, Al-Mousa R, Sundar G, Lim TC. mydriasis during orbital floor fracture reconstruction: a novel diagnostic and treatment algorithm. Craniomaxillofac Trauma Reconstr 2010;3:209-16.
3. Goodwin IA, Stotland MA. Transient oblong anisocoria and orbital surgery. J Craniofac Surg 2012;23:2011-3.

4. Newell FW. Ophthalmology: principles and concepts. St. Louis: The C. V. Mosby Company; 1965.

5. Lee JM, Kim CH, Kim UK, Chung IK. Transient ipsilateral mydriasis during correction of left blowout fracture. J Craniofac Surg 2014;25:527-8. 